\title{
El incipiente reconocimiento de la ciudad histórica ante la llegada de la ciudad industrial: del siglo xIx e inicios del $x x$
}

The incipient recognition of the historic city before the arrival

of the industrial city: from the 19th century

and the beginning of the 20th century

Luz Cecilia Rodríguez Sánchez
http://orcid.org/0000-0002-1546-9050

Primera versión recibida en: 05 mayo, 2019

Última versión recibida en: 27 septiembre, 2019

\section{Resumen}

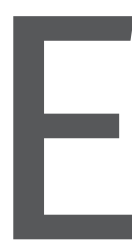

I objetivo de este artículo reside en explicar de qué manera se dio el reconocimiento de la ciudad histórica a finales del siglo XIX y principios del XX, a través de un estudio historiográfico, a fin de comprender que dicho reconocimiento obedece a circunstancias muy específicas, más allá de una va-

1 Arquitecta y maestra en Conservación del Patrimonio Cultural por la Universidad Autónoma Metropolitana, doctora en Historia Moderna y Contemporánea por el Instituto de Investigaciones Dr. José María Luis Mora; Miembro del Sistema Nacional de Investigadores (sni) del Consejo Nacional de Ciencia y Tecnología (Conacyt); Profesora-investigadora de tiempo completo en la Facultad de Arquitectura "5 de Mayo" de la Universidad Autónoma "Benito Juárez" de Oaxaca: Líneas de investigación: espacio público, regeneración urbana, centros historicos e historia urbana. loración estética, pues detrás de ella está un discurso referente a la controvertida llegada de la modernidad y el ideal del progreso que permeó el siglo XIX, lo que se verá reflejado en la forma en cómo se concibe la ciudad, y en la generación de diversos proyectos de intervención urbana, que serán los detonantes para que cierto grupo de intelectuales advierta las amenazas generadas por la transformación urbana que acompaña al discurso de la modernidad. Asimismo, se hace referencia al caso específico de la intervención de Hausmmann, a la ciudad de París, como un detonante que, si bien fue severamente criticado por los conservadores, se convierte en un parteaguas en el proceso de reconocimiento de la ciudad histórica. En esencia, este trabajo busca presentar un escenario más horizontal respecto al génesis de la valoración de las ciudades históricas, para vislumbrar de una forma más integral cómo se gestó dicho proceso y, sobre todo, entender que las acciones y los juicios emitidos

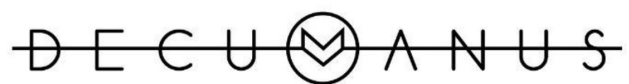

REVISTA INTERDISCIPLINARIA SOBRE ESTUDIOS URBANOS 
por diversos personajes de la época fueran fruto de un periodo histórico específico, razón por la cual deben ser juzgados a partir de una comprensión más amplia del pasado.

Palabras clave: ciudad antigua, conservación urbana, historia urbana

\section{Abstract}

The aim of this article is to explain how the historic city was recognized at the end of the 19th century and the beginning of the 20th, through a historiographic study, in order to understand that this recognition obeys very specific circumstances, beyond an aesthetic assessment, behind it is a discourse referring to the controversial arrival of modernity and the ideal of progress that permeated the 19th century, which will be reflected in the way in which the city is conceived, and in the generation of various projects of urban intervention, which will be the triggers for a certain group of intellectuals to notice the threats generated by the urban transformation that accompanies the discourse of modernity. Likewise, reference is made to the specific case of Hausmmann's intervention, the city of Paris, as a detonator that, although severely criticized by conservatives, becomes a watershed in the process of recognition of the historic city. In essence, this work seeks to present a more horizontal scenario with respect to the genesis of the valuation of historic cities, to glimpse in a more integral way how this process was conceived and, above all, to understand that the actions and judgments emitted by diverse characters of the time were fruit of a specific historical period, reason for which they must be judged from a broader understanding of the past.

\section{Keywords}

Ancient city, urban conservation, urban history

\section{Introducción}

Desde finales del siglo $x x$ el concepto de centro histórico comenzó a ser un término empleado más allá del ámbito de la conservación, hasta convertirse en una expresión utilizada cotidianamente por la sociedad; no obstante, pocas veces se reflexiona acerca del origen que tiene el reconocimiento de la ciudad histórica, como parte no solo del proceso urbano, cuyo origen reside en la fundación de un asentamiento humano, al que se suman varias transformaciones, tanto físicas como funcionales y perceptuales. Asimismo, dicho reconocimiento obedece a una serie de factores que involucran otras variables, como es el cambio de pensamiento y la confrontación de ideologías, las cuales provocaron enfrentamientos entre los promotores de la modernidad y los conservadores.

Con base en lo anteriormente expuesto, este texto se divide en cuatro partes. En la primera se aborda de forma general, a modo de preámbulo, el proceso previo que dio origen al reconocimiento del patrimonio urbano; en segundo término, se ahonda en la confrontación ideológica que se genera en torno a la modernidad y al progreso que acarrea consigo la Revolución Industrial, la cual se con-

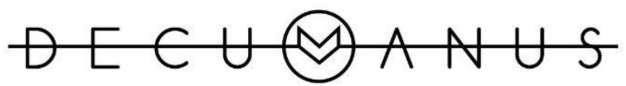

REVISTA INTERDISCIPLINARIA SOBRE ESTUDIOS URBANOS 
vierte en detonante para la transformación de las ciudades y, por ende, representa una amenaza para las ciudades históricas. En un tercer apartado se hace una revisión de los diferentes discursos que comenzaron a surgir durante el siglo XIX y principios del $x X$ respecto al reconocimiento de la ciudad histórica a fin de identificar cómo fue madurando y enriqueciéndose dicho discurso, que se convertirá en la base de la conservación urbana. Posteriormente se alude del caso específico de la intervención urbana de Haussmann, guiada por los ideales de la modernidad.

Por último, se concluye con una serie de reflexiones a fin de subrayar la importancia de contar con una lectura más de carácter horizontal, en cuanto al hecho de comprender que el proceso de reconocimiento de la ciudad histórica es producto de un momento histórico, cuyas condiciones y formas de pensar sentaron las bases para su consolidación.

En ese sentido, el estudiar y analizar el modo en que se fue presentando este proceso de reconocimiento de la ciudad histórica obedece a la necesidad de entender de una manera más integral cuáles fueron sus orígenes, pues más allá de las cartas y documentos internacionales emitidos en el siglo Xx, tales como la carta de Atenas y la carta de Venecia, junto con otras más, existe una historia previa que da origen al reconocimiento de la ciudad histórica. Así pues, para comprender cómo fue dicho proceso, es necesario ahondar en el pensamiento de la época que provocó un singular enfrentamiento entre los conservadores y los modernistas, para poder establecer relaciones entre diferentes procesos que, de manera directa o indirecta, impactaron en la valorización de la ciudad antigua y en el establecimiento de las primeras pautas dirigidas a su conservación.

La metodología empleada está basada en una revisión documental de fuentes primarias y secundarias dividida en tres etapas -búsqueda, sistematización y análisis-. Cabe señalar que no fue un proceso lineal, sino que, al trabajar de manera interdisciplinaria, el mismo proceso de análisis condujo a nuevas búsquedas para ir dando coherencia al texto a lo largo de su desarrollo. Asimismo, es pertinente mencionar la importancia que tuvo la revisión de fuentes primarias, pues muchas de las obras, aunque han sido traducidas al castellano, en ocasiones se identificaron pérdidas del sentido original en la traducción. La selección de las obras consultadas se planteó desde un inicio con carácter interdisciplinario, en el sentido de comprender un espectro más amplio que el ofrecido por el urbanismo y la conservación urbana, a fin de indagar sobre el pensamiento de la época a través de la revisión de diversos pensadores y obras literarias.

\section{La identificación de la ciudad histórica: breviario historiográfico}

Si bien las transformaciones que sufren la ciudades en el siglo XIX se atribuyen a los efectos de la Revolución Industrial, la conceptualización de transformar la ciudad en un espacio racional y geométrico, es resultado de

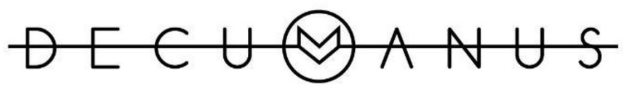

REVISTA INTERDISCIPLINARIA SOBRE ESTUDIOS URBANOS 
la mentalidad del Renacimiento y posteriormente de la Ilustración, periodo en el cual se produce una revalorización de la cultura clásica en contraposición al oscurantismo medieval (Pérez Blázquez, 2010). Asimismo, con el surgimiento del humanismo, el cual buscaba desvincular al hombre de la dependencia divina, a partir de concebir al ser humano como un ser libre, se produce un rompimiento con la ideología de la Edad Media, caracterizada por el teocentrismo. Dicho rompimiento con el pasado inmediato tiene como resultado la resurrección de la antigüedad, que despertó un notable interés por el periodo clásico, tanto en lo arqueológico como en lo literario. Surge el florecimiento de la llamada burguesía, y se produce la decadencia del feudalismo (M. González, 1994).

Este interés y admiración por la cultura clásica fue resultado de una necesidad ideológica, en tanto que la burguesía, como lo señala Karl Marx, se ve obligada a recurrir a los ideales de la República Romana para ocultar "el contenido burguesamente limitado de sus luchas y mantener su pasión a la altura de la gran tragedia histórica" (Marx, 2003, p. 11), ciertamente, este acto puede considerarse como una negación del pasado inmediato, lo que se traduce en pretender recobrar un pasado anterior, con el fin de determinar qué es digno de admirar o valorar $y$, por tanto, de considerarlo monumento, como lo fueron en su caso las ruinas romanas. Esto equivale a una resignificación de los vestigios del pasado como elementos dignos de ser valorados y resguardados.

Así pues, el Renacimiento constituye un periodo relevante en la historia de la conservación, al mismo tiempo, se manifiesta el reconocimiento del monumento de una manera más evidente, puesto que se establece una diferenciación entre los monumentos artísticos y los históricos, que se mantendrá hasta el siglo XIX (Rielg, 1987, p. 36).

La revolución de pensamiento no se mantuvo ajena a la visión en cómo era concebida la ciudad, René Descartes (1596-1650) pensador de la época, preocupado por el naufragio del llamado pasado filosófico, manifiesta en el Discurso del Método, su crítica al desorden que permeaba a las ciudades antiguas, "Esas viejas ciudades que no fueron al principio sino aldeas, y que, con el transcurso del tiempo han llegado a ser grandes urbes, están, por lo común, muy mal trazadas y acompasadas, si las comparamos con estas otras plazas regulares que un ingeniero diseña, según su fantasía, en una llanura [...]" (Descartes, 2010, p. 41). Este fragmento muestra la esencia de un pensamiento y de una manera de ver la realidad bajo una concepción que rechaza aquello que parece irracional, o morfológicamente irregular, lo cual se convertirá en un referente un tanto utópico para la época respecto al deber ser de la ciudad. Durante el siglo XVII y XVIII dichos planteamientos serán retomados para el trazo de las ciudades, cuya finalidad era reflejar el orden y racionalidad (Layuno, 2013).

Ahora bien, la postura crítica hacía la irregularidad de las ciudades puede ser identificada como un hecho previo a la Revolución Industrial, aunque no se materializa bajo una reforma urbana como tal, sí se convierte en un ideal del deber ser de esta, que alcanzará una etapa de maduración en el pe-

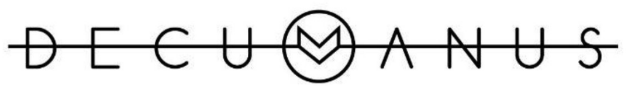

REVISTA INTERDISCIPLINARIA SOBRE ESTUDIOS URBANOS
Núm. 4. Vol. 4. Octubre 2018-Octubre 2019. Instituto de Arquitectura, Diseño y Arte.

Universidad Autónoma de Ciudad Juárez. ISSN: 2448-900X 
riodo barroco, en el que se busca una mayor regularidad en la traza urbana, con grandes espacios abiertos, que favorezcan los campos visuales, por lo que la ciudad medieval constituida a través de una traza tortuosa impedía que las fachadas y los monumentos fuesen admirados.

Cabe señalar que durante el siglo XVII, la Guerra Civil europea inició un amplio movimiento de restauración después de la destrucción, pues el reconocimiento del monumento y la valorización de los edificios antiguos fue resultado del emergente coleccionismo de antigüedades que estaba surgiendo en el mundo occidental, especialmente en aquellos países involucrados en la lucha y el expansionismo territorial (Zeayter y Mansour, 2018).

El urbanismo barroco, por lo tanto puede ser leído como una continuidad del Renacimiento italiano, al decantar los principios de este y al servirse de la línea recta para uniformizar la traza, demoler edificaciones que consideraba carentes de valor estético o que implicaban un estorbo para dar lugar a las amplias avenidas cuyo remate fuese una construcción emblemática o un monumento (Fernández Christlieb, 1998). Esta visión crítica de la ciudad, representa un anhelo por racionalizar el espacio, no se traduce en un deseo voraz por eliminar la ciudad antigua, pues estos ideales de orden y simetría se verán concretados más tarde en las primeras ampliaciones de las ciudades o en la edificación de nuevas áreas urbanas y colonias, resultado de los procesos de industrialización que surgen durante los siglos xIX y xx (Layuno, 2013).
En esta etapa previa a la llamada conservación moderna del patrimonio, aunque no hay un evidente reconocimiento del patrimonio urbano, entendido este como conjunto, es cuando comienza a darse una identificación del monumento en singular, primero bajo el reconocimiento de los edificios antiguos producto de un sentimiento romántico, que encuentra en las ruinas un encanto particular, al grado de llegar a recrear un aspecto de abandono, como fue el caso de Kassel en Alemania, en donde se edificó un nuevo castillo en ruinas, al mismo tiempo que se buscaba mantener en uso los viejos castillos mediante la instauración de programas de reparación, bajo la consigna de conservar aquello que fuera antiguo, como sucedió en Escocia a finales del siglo XVIII (Glendinning, 2013).

El caso de Escocia resulta relevante debido a que el reconocimiento por lo antiguo, que se produjo en esta nación, dio origen a un paisaje salpicado de múltiples castillos ruinosos, lo que a su vez generó el concepto de "paisaje histórico escocés", mismo que se vería reflejado en obras literarias como lo fueron los poemas de James Macpherson, creador del mito de Ossian que alude a dichos paisajes. Asimismo, esta nostalgia por lo antiguo también tiene una raíz en el incipiente nacionalismo que comienza a permear el territorio europeo (Glendinning, 2013).

Así pues, dicha sensibilidad hacia lo antiguo que emerge durante el siglo XVIII puede ser vista como un acto más de carácter sentimental o amateur. Sin embargo, a finales de siglo las revoluciones ideológicas, sociales y económicas serán el motor para conformar

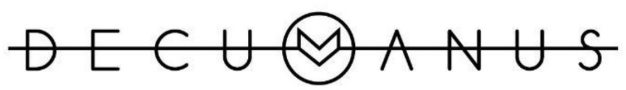

REVISTA INTERDISCIPLINARIA SOBRE ESTUDIOS URBANOS 
un verdadero movimiento conservador de los monumentos históricos.

Es importante hacer notar que la Revolución Industrial va a ser un punto clave dentro de la historia de este naciente movimiento, ya que trajo consigo una serie de cambios acelerados que trastocaron a la sociedad de muy diversas formas; las ciudades, por lo tanto, fueron las primeras en manifestar los sintomas de los cambios sociales y económicos que dieron lugar a la transformación de las formas de producción que se tradujeron en el aumento de la producción industrial y en el crecimiento y multiplicación de los intercambios mercantiles, lo que desató la problemática urbana. Dicho fenómeno es denominado por Henry Lefebvre como implosión-explosión: "Concentración urbana, éxodo rural, extensión del tejido urbano, subordinación completa de lo agrario a lo urbano" (Lefebvre, 1988, p. 473). Este proceso de urbanización que se da durante la primera Revolución Industrial, misma que provoca la reorganización del espacio (Castells, 2004, p. 21), a consecuencia de una descomposición de las estructuras sociales (emigración de la población rural a zonas urbanas) que darán lugar a una modificación del paisaje urbano alterando sus estructuras, y agregando nuevos elementos que convivirán o sustituirán a los preexistentes.

\section{Confrontación ideológica entre el progreso y la conservación}

Con la llegada de la Revolución Industrial, las ciudades comienzan a sufrir diversas alteraciones, que se evidencian en su funcionamiento y su morfología. Es importante destacar que este fenómeno se experimenta en muy diversos niveles, puesto que la sociedad occidental atraviesa por un proceso de transformación y adaptación provocado por la industrialización, y aunque dicho fenómeno tiene un origen económico y tecnológico, también va a estar acompañado y respaldado por un discurso ideológico que debe ser tomado en cuenta para comprender de manera integral de dónde surge esta necesidad de conservar y reconocer el valor de la ciudad histórica.

En el siglo XIX parte del discurso intelectual giró en torno al significado de ser moderno y de la búsqueda del llamado progreso, lo que repercutirá en diferentes ámbitos, puesto que los avances tecnológicos se verán reflejados a diversas escalas. De ahí que lo llamado moderno comience a ser un anhelo que busca desplazar al pasado y a lo tradicional, fenómeno que tendrá eco en el ámbito de lo urbano.

En este sentido, David Harvey, conceptualiza al pensamiento de la modernidad como aquel que no tiene respeto por el pasado, es por ello que la conservación representa un opuesto a la misma, en cuanto a que la modernidad avanza en sentido contrario a la tradición, mientras que por otra parte la propia modernidad se ve impulsada por un ideal de transformación, de crecimiento y de progreso, de ahí la referencia que hace Harvey y Marshall Berman a Karl Marx, sobre el significado del ser moderno, pertenecer a un universo en el que todo lo sólido se disuelve en el aire; esta metáfora responde a la condición moderna definida por lo efímero y el constante cambio caótico (Harvey, 1998, pp. 24-25). De ahí que la condición de

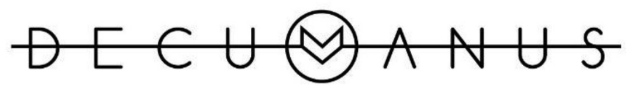

REVISTA INTERDISCIPLINARIA SOBRE ESTUDIOS URBANOS 
la modernidad abogue por la ruptura respecto a todas aquellas condiciones históricas precedentes, por lo que dicho proceso se puede abordar como una fragmentación que se antepone a la consolidación de una continuidad.

Es pertinente hacer notar que bajo esta corriente de pensamiento surgirá una contradicción ideológica, respecto a si la modernidad es concebida como progreso, innovación y vanguardia, que para lograr materializarse se hace valer de la ruptura con lo establecido, lo que provocará la conceptualización de la llamada destrucción creadora, cuyo origen retórico es en esencia un oxímoron, puesto que se construye a partir de una dualidad incoherente, si se parte de un principio tradicional en donde construir es crear y destruir es eliminar. No obstante, la llamada destrucción creadora se sustenta en que a partir del momento en que la modernidad es llevada a la práctica, y se materializa a través de diversos proyectos tangibles, para poder ser, tiene que destruir o desaparecer aquello que le precedió, “cómo se crearía un mundo nuevo sin destruir gran parte de lo ya existente?" (Harvey, 1998, p. 31). Dicha analogía surge de "la suposición de que a través de la destrucción de la estructura urbana preexistente se consigue la regeneración generalizada del ambiente urbano" (Sánchez de Juan, 2000). Ahora bien bajo esta misma noción, al comparar el proceso de renovación urbana generado por los efectos de la Revolución Industrial, se puede establecer al mismo tiempo un vínculo con la teoría económica de Joseph Alois Schumpe- ter (1883-1950), ${ }^{2}$ quien sostenía que la esencia del capitalismo era la innovación permanente, al considerar que el motor de la economía reside en la constante mutación industrial, generada a partir de la continua destrucción de lo antiguo para dar lugar a nuevos elementos, de ahí su frase que define a este como "el vendaval perenne de la destrucción creadora" (Schumpeter, 1996, p. 121).

Será entonces bajo esta noción de destrucción creadora que se puede dar una lectura a los cambios urbanos que sufrieron las ciudades históricas, en donde la imposición del arribo de la ciudad moderna pretenderá imponerse sobre las estructuras de la ciudad histórica, pues bajo la ideología de la época, este deseo de sustitución de las antiguas estructuras urbanas por nuevos modelos, va más a allá de un fenómeno de carácter estético, debido a que la preocupación por intervenir la ciudad a fin de hacer de ella un espacio más sano, funcional y atractivo va a responder a la suma de intereses de carácter económico, político y social.

Sin embargo, el pensamiento de cada época dista de ser homogéneo, y menos aún cuando la sociedad envuelta en este proceso de cambio perpetuo genere opiniones diversificadas, de ahí que el principio de la destrucción creadora sea solo uno de los puntos de anclaje para comprender dicho desarrollo de renovación

2 La noción de destrucción creadora, si bien se atribuye a la teoría económica de Schumpeter, esta estuvo influenciada por las obras de Goethe (Fausto) y de Nietzsche (Así habló Zaratustra), y por la obra de Werner Sombart, quien explica que el origen del capitalismo moderno obedece a la destrucción de los antiguos modelos económicos (de la Corte Carmona, 2015). 
urbana, que en primera instancia implicaba un atentado contra aquello que fuera preexistente. La imagen histórica concebida respecto a este periodo, se caracteriza por la sed de progreso que permeaba a la sociedad, entendida esta como una disputa entre antiguos y modernos, "el momento en el que los europeos occidentales se tomaron la libertad de creer que habían superado a la antigüedad, que el Renacimiento había terminado por completo y que había nacido la religión del Progreso" (Gellner, 1997, p. 116), no significa que haya sido un pensamiento generalizado dentro de la sociedad y menos aún respecto a los pensadores de la época.

Ciertamente, hubo quienes se vieron segados por la modernidad prometedora, pero de forma sincrónica también coexistió una visión romántica que rechazaba la postura progresista de los llamados modernos con relación a su pensamiento determinista y acrítico referente al pasado. Entre quienes condenaban aquello que significara abolir al pasado, hubo tanto románticos radicales que repelían lo que fuese moderno, como moderados que abogaban por el reconocimiento de una continuidad al identificar "las semillas del presente en el pasado, la realización del pasado en el presente" (Gellner, 1997, p. 117) y sobre todo son los que le darán un valor y una orientación diferente a la historia, puesto que sin este pensamiento difícilmente se podría entender el sentido de la conservación.

Los contrapesos ante este escenario van a ser justo los motores del movimiento conservador moderno, como respuesta hacia aquellos que preten- dían extinguir el pasado, dado que a este se le confiere y se le reconoce un significado y un sentido de ser para la sociedad a la cual pertenece. El siglo XIX, permeado por las corrientes nacionalistas va a promover la confrontación entre viejas y nuevas identidades, lo que conlleva a recurrir a diversas "doctrinas, sistemas de símbolos y representaciones, entre ellas las patrimoniales" (Prats, 2000, p. 122), que darán lugar a la llamada edad de oro del patrimonio nacional en Europa.

\section{El reconocimiento de la ciudad histórica ante el amenazante arribo de la modernidad urbana}

Ante los continuos cambios que trajeron las diversas innovaciones tecnológicas, algunas ciudades comenzaron a ver trastocada su fisionomía, al mismo tiempo que aquellos que abogaban por la modernidad vieron en las ciudades un basto campo de trabajo para poner en práctica los ideales de progreso.

La llegada de nuevos materiales y sistemas de construcción (acero, concreto y vidrio) serán elementos determinantes en la transformación de la escena urbana, pues surgen nuevos edificios que dominarán en altura a sus antecesores, lo cual produce de forma paralela una mayor densificación del tejido urbano. A ello se suma la llegada del tranvía y del metro en la segunda mitad del siglo XIX $y$, posteriormente en las primeras décadas del siglo xx, la proliferación de los automóviles, elementos que serán precursores de la expansión urbana, cuyos

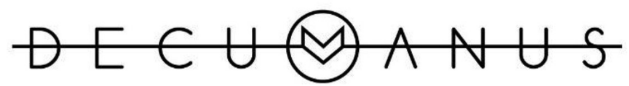

REVISTA INTERDISCIPLINARIA SOBRE ESTUDIOS URBANOS 
efectos también se verán en la imagen de la ciudad (Choay, 2009).

Durante la primera mitad del siglo XIX se dan ciertas manifestaciones en el quehacer de la historia urbana, las cuales muestran el interés por el estudio de la forma, mediante críticas románticas a la ciudad, lo que para mediados de siglo se traducirá en el surgimiento de diversas corrientes dirigidas a la conservación de monumentos que estaban siendo amenazados (Capel, 2002, p. 45). Si bien se reconoce el valor de edificaciones particulares, habrá quienes comiencen a apreciar, no solo la singularidad del monumento, sino la conformación del conjunto en el cual se encuentran insertas dichas edificaciones, producto justo de esta sensación de amenaza evidente que representaba la industrialización, que no solo se estaba expresando como un fenómeno de expansión urbana, sino también de transformación y adecuación de la ciudad pretérita.

Aunque el movimiento de conservación de monumentos tiene su origen en los individuales, poco a poco fue expandiéndose al nivel urbano hasta llegar a comprender ciudades antiguas enteras. Entre los primeros ejemplos se encuentra la ciudad alemana de Nuremberg, que en el siglo xVIII había sido considerada por los viajeros como un lugar viejo, atrasado y repulsivo. No obstante, a finales del siglo XVIII Wilhelm Heinrich Wackenroder (1773-1798) junto con Ludwig Tieck (1773-1853) publicaron un manifiesto de carácter romántico y nacionalista que enaltecía a la ciudad; en poco tiempo dicho texto se convirtió en una de las obras fundacionales del roman- ticismo alemán, que se tradujo en un llamado por conservar la ciudad, misma que en algún momento fue la residencia de Durero. En dicho manifiesto se hacía alusión al estado de conservación que lograba mantener la apariencia antigua de la ciudad, gracias a que no se había construido ningún edificio nuevo (Glendinning, 2013).

El texto de Wackenroder sin duda fue de gran trascendencia, pues durante el siglo XIX y principios del xx emergió el culto hacia las ciudades antiguas, lo cual se verá respaldado por la obra de Goethe (1749-1832), Hermann y Dorothea, que idealiza las ciudades pequeñas versus su contraparte, las incipientes ciudades industriales. Bajo este mismo enfoque se encuentra la obra de Welby Pugin (1812-1852), Contrastes, publicada en 1836 , en la que enaltece la arquitectura medieval frente a la decadente arquitectura moderna, a través de una comparación entre la excelencia de las edificaciones católicas con la supuesta degeneración moderna (Pugin, 1898).

Para finales del siglo XIX la conservación aislada de monumentos se vio superada por la valoración de los conjuntos urbanos, esto se tradujo en una apreciación sin precedentes hacia la ciudad antigua, lo que llevó a establecer la moda de anteponer el prefijo de viejo al nombre de las ciudades, Alt-Nürnberg (viejo Nuremberg), Vieux-Paris (viejo París), es así como se delata el reconocimiento de la escala urbana y paisajística.

Esta ferviente admiración hacia los cascos antiguos responde a los cambios que empiezan a sufrir las ciudades. Asimismo, el enaltecimiento de las ciudades medievales, si bien es

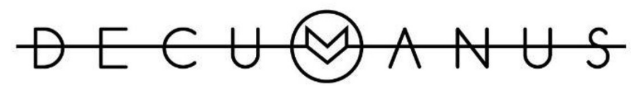

REVISTA INTERDISCIPLINARIA SOBRE ESTUDIOS URBANOS 
resultado de un reforzamiento del nacionalismo, también se vio acompañado por el crecimiento del turismo.

La consolidación del reconocimiento al valor urbano se traduce en la creación de un nuevo término, Altstadt, que en alemán significa "casco antiguo", reflejo de la importancia que toma la ciudad fundacional respecto a su contraparte modernizadora. El empoderamiento que adquiere el movimiento de conservación se traduce en la apreciación de la arquitectura de acompañamiento, a decir de Glendinning, los efectos fueron evidentes, pues "de ahora en adelante, los edificios 'de fondo' ocuparon el primer plano de atención" (Glendinning, 2013), esto en sí constituye el principio de la conservación urbana, al dejar de ver el monumento aislado, sino el conjunto de una manera más integral.

Las manifestaciones que tendrán estas corrientes por la conservación de la ciudad histórica se presentarán en diferentes matices y latitudes, como una reacción ante aquellos que consideraban las estructuras urbanas heredadas como elementos obsoletos (Díaz Berrio, 2007, p. 23), y por tanto, sustituibles por nuevas estructuras modernas. Estas expresiones comenzarán a tener lugar en Europa, encabezadas por diversos personajes con diferentes procedencias y opiniones, en Gran Bretaña John Ruskin y William Morris; en Francia, Viollet le Duc; en Italia, Camillo Sitte y más tarde Gustave Giovanonni; en Alemania, Patrick Geddes y Joseph Stübben, entre otros.

Este nutrido encuentro de opiniones dio lugar a múltiples discusiones en torno a cuál debía ser el futuro de las ciudades históricas, si bien estas en principio se mantuvieron bajo un escenario teórico, fueron ideas que a su vez dieron lugar a diversas obras que hoy en día son parte fundamental de la historia, no solo de la conservación sino del urbanismo como disciplina, serán también las voces que en las primeras décadas del siglo xx, construirán el andamiaje de la conservación urbana mediante la elaboración de principios, teorías, prácticas, experiencias, herramientas técnicas y normativas dirigidos a preservar el patrimonio urbano (Bandarin y van Oers, 2012).

Para entender cómo se fue gestando este discurso respecto a la identificación y valoración del patrimonio urbano, a continuación, se presenta una revisión general de las diferentes posturas a fin de comprender por qué se reconoció el valor de la ciudad histórica.

La conservación de monumentos $y$, por ende, de ciudades, no es una historia que deba ser leída al margen de la que corresponde a la modernidad que permeó el siglo $\mathrm{XIX}$, sino que es una historia paralela que se va moldeando de acuerdo con las circunstancias y a los acontecimientos de su época. Los sucesos ocurridos en torno a la conservación deben leerse como un espejo de los efectos de la modernidad y como una reacción a la corriente dominante: lo viejo frente a lo nuevo, lo estático frente a lo dinámico, lo mixto frente a lo segregado, etcétera. Sin embargo, en ocasiones esta postura de oposición entre los arquitectos modernos y los líderes de la conservación estaban de acuerdo en la necesidad de una segregación absoluta y la legibilidad de lo nuevo y lo viejo, mientras que en otros momentos el

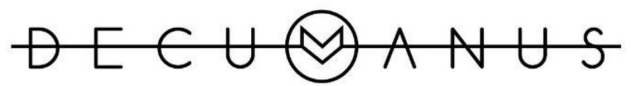

REVISTA INTERDISCIPLINARIA SOBRE ESTUDIOS URBANOS 
mismo movimiento de conservación reprodujo directamente los valores modernos más tajantes, como la moda adoptada de aislar los monumentos, demoliendo todo aquello que los rodeaba, o el agudo "valor de novedad" de las restauraciones radicales lideradas por Viollet Le-Duc (Glendinning, 2013). Asimismo, la vasta campaña de restauración de monumentos desempeñó un papel vital en la agudización del nuevo sentido histórico del futuro nacional basado en el patrimonio.

A lo largo del siglo XIX, una vez reconocido el valor patrimonial de ciertos edificios, surgieron diversas opiniones respecto a cómo debían ser tratados, que llevó a la confrontación de dos defensores de los monumentos, por un lado, viollet Le-Duc con su teoría restauradora y por otro, Ruskin, con su postura conservadora.

Eugène-Emmanuel Viollet Le-Duc (1814-1879) publicó, en 1866, su Diccionario razonado de arquitectura, en el que argumentó que la "palabra y el significado son modernos, restaurar un edificio, no es mantenerlo, arreglarlo o rehacerlo; es restablecerlo en un estado completo que puede no haber existido nunca" (Le-Duc, 1995, p. 15). Por lo tanto, para Viollet Le-Duc, la restauración de un edificio equivalía a la reconstitución de un estado completo e ideal, al grado de tener una apariencia que jamás tuvo. Bajo este enfoque fue que Le-Duc intervino no solo monumentos individuales, entre otros Notre Dame en París, sino también complejos urbanos, como fue la polémica restauración y la reconstrucción de la ciudad francesa de Carcassonne (Bandarin y van Oers, 2012). Dentro de su visión, él comprendía al monumento como una unidad, lo que lo llevó a entenderlo como un objeto aislado, esto lo mantenía ajeno a su relación con el entorno y a su valor como elemento urbano (Capitel, 1988).

Con base en lo mencionado anteriormente, la llegada de la industrialización a las ciudades representó una amenaza directa a sus construcciones, despertando la atención de algunos pensadores y arquitectos de la época. Uno de los primeros en levantar la voz al respecto, fue Jonh Ruskin (18191900) quien consideraba que la magnificencia de una ciudad no radicaba en la belleza de las grandes construcciones, sino en la unidad que conforman como conjunto, el que también estaba integrado de edificaciones menores (Capitel, 1988). Este pequeño guiño representa uno de los primeros pasos para reconocer el valor del conjunto $y$, por ende, del patrimonio urbano, al concebir "el tejido como el ser de la ciudad y como un objeto patrimonial intangible que tiene que ser protegido incondicionalmente" (Choay, 2007, p. 145), lo que se traduce en la identificación del conjunto como monumento colectivo, silenciosamente análogo al antiguo monumento conmemorativo (Glendinning, 2013).

Bajo este tenor, Ruskin y William Morris (1834-1896) reaccionaron ante la modernidad amenazante que comenzaba a atentar contra los edificios históricos y, en consecuencia, a la destrucción y alteración del patrimonio ambiental que representaban las ciudades históricas de Gran Bretaña (Tomaselli, 2013). Tanto Ruskin como Morris definirán su postura en torno al reconocimiento del valor de los edificios y sitios históricos; sin embargo, se

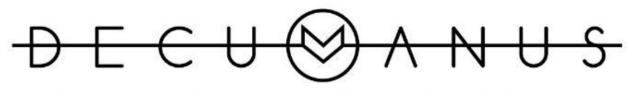

REVISTA INTERDISCIPLINARIA SOBRE ESTUDIOS URBANOS 
oponían a los trabajos de restauración que modificaran la esencia de estos, de ahí la expresión de Ruskin "Que no se hable de restauración. Es una Mentira de principio a fin" (Ruskin, 1996, p. 173), dado que él se resistía a la recreación del pasado, pues toda intervención se traducía en una alteración a la esencia original, al considerar la restauración como sinónimo de destrucción (di Montauto, 2003, p. 440).

A partir de este principio, Ruskin criticó a los restauradores por la destrucción de la autenticidad histórica de los edificios y abogó por la protección, la conservación y el mantenimiento que no alterara la esencia de los monumentos. Mientras que William Morris, como líder de la Sociedad para la Protección de Edificios Antiguos (SPAB, por sus siglas en inglés) sostenía de manera metafórica que para evitar la caries se debía procurar el cuidado diario.

Entre los referentes fundamentales de este periodo se encuentra Camillo Sitte (1843-1903), quien en su obra Construcción de ciudades según principios artísticos, publicada en 1889, resalta la inigualable relevancia de las ciudades antiguas, que deben ser analizadas y estudiadas para poder elaborar propuestas racionales acordes a la ciudad moderna, procurando salvar de la destrucción a las ciudades antiguas (Sitte, 1926, p. 3), por lo que debe encontrarse un balance entre la técnica y la estética. La defensa que plantea Sitte respecto a la ciudad antigua, si bien obedece a un reconocimiento de su valor artístico, también tiene un carácter funcional, en virtud de que las ciudades preindustriales constituyen modelos de referencia para las ciudades modernas. Entre las principales aportaciones de Sitte, se encuentra el presentar una noción más amplia del paisaje, al ir más allá del mero objeto arquitectónico e involucrar a los diferentes elementos que conforman el conjunto, como son los espacios abiertos y las plazas.

A decir de Glendinning, no debe olvidarse que la intención de Sitte era el generar principios para la construcción de nuevas ciudades, dado que él consideraba que el urbanismo moderno era demasiado geométrico, formulista, materialista y burocrático (Freestone, 2007). Es importante resaltar que el trabajo de Sitte influenció a varios pensadores de la época, tales como Alois Riegl en Austria, Josef Stübben en Alemania y Charles Buls en Bélgica, quien abogó por la planificación sensible y respetuosa.

En el caso específico de Josef Stübben (1845-1936), en su obra Construcción de ciudades, publicada en 1890 , hace énfasis respecto a las ciudades antiguas, pues si bien muestra una clara preocupación por su adaptación a las necesidades de la modernidad, ve en las trazas medievales un carácter pintoresco, justo resultado de sus irregularidades, por lo que se opone a la geometrización de las trazas, pues ello llevaría a perder "inigualables escenas callejeras, cuya pérdida debe ser evitada por la modernidad" (Stübben, 1907, p. 189).

Asimismo, Stübben identifica y establece una clara diferenciación entre centro y periferia, al mencionar que uno de los componentes principales de la metrópoli es el "El centro de la ciudad o casco antiguo, cuyo centro suele ser llamado el corazón de la

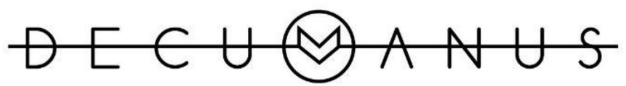

REVISTA INTERDISCIPLINARIA SOBRE ESTUDIOS URBANOS 
ciudad" (Stübben, 1907, p. 57), tal diferenciación delata, por un lado, el reconocimiento de las características históricas y monumentales de una parte de la urbe $y$, al mismo tiempo, el crecimiento de la misma que llevó a la conformación de nuevas zonas con propiedades y comportamientos totalmente diferentes. De ahí que Stübben al tener presente que las ciudades antiguas debían adaptarse a ciertas necesidades de la época, partiera de que las adaptaciones tendrían que realizarse siempre respetando la individualidad del lugar.

También dentro del marco alemán, se encuentra Patrick Geddes (18541932), quien manejó una visión cercana a la de Sitte, de acuerdo con la apreciación visual y estética de las ciudades, en la que concebía a la urbe como un organismo en evolución, donde los componentes físicos y sociales interactuaban bajo un sistema de continua transformación y tradición, equiparable con un organismo vivo, lo cual puso en evidencia su formación como biólogo, al retomar una visión naturalista adaptada al ámbito urbano, puesto que él concebía la ciudad como un ente en constante adaptación, en el que cada generación era capaz de aportar un cambio físico al espacio a través de la creación y transformación de nuevas estructuras y funciones. Este pensamiento se verá reflejado en su obra Ciudades en evolución (1915), en donde reconoce a la ciudad histórica como elemento fundamental para comprender el funcionamiento de las urbes, así como el valor de las huellas, los recuerdos y las asociaciones colectivas de determinados espacios, y poder emprender los procesos de transformación urbana, a partir del reconocimiento y respeto a los mismos.

Geddes formuló una visión integral de la urbe, de ahí que identificó la importancia de la ciudad antigua como parte fundamental para su desarrollo. Bajo este enfoque acuñó el término de conservación quirúrgica como la base de la conservación urbana a fin de reducir al mínimo la destrucción de los edificios y espacios históricos con la intención de adaptarlos a las necesidades de la modernidad (Zeayter y Mansour, 2018).

Entre otros visionarios de la ciudad histórica, se encuentran Werner Hegemann (1881-1936) quién en 1922 publicó junto con Elbert Peets (18861968) El Vitrubio americano, en el que retoma el llamado método analítico de Camillo Sitte, para demostrar la importancia que tienen en las ciudades los elementos de continuidad como parte de un proceso armonioso de desarrollo. Hegemann concebía la ciudad como un gran collage que estaba en permanente transformación, cuya identidad y significado dependía de la interacción de todas sus partes (Bandarin y van Oers, 2012).

Durante las primeras décadas del siglo Xx, la discusión referente al diálogo que debía mantener la ciudad antigua respecto a la ciudad moderna comenzará a ser cada vez más recurrente y sistematizado; uno de los ejemplos más relevantes es la obra de Gustave Giovannoni (1843-1947), al reconocer el valor del tejido urbano como parte esencial del testimonio histórico y cultural, que debía ser protegido. Cabe señalar que Giovannoni se formó como ingeniero y se especializó en la escuela higienista y también como historiador

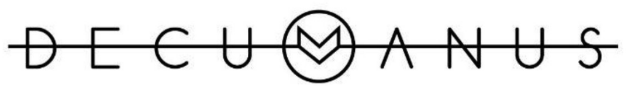

REVISTA INTERDISCIPLINARIA SOBRE ESTUDIOS URBANOS 
del arte, lo que le brindó la sensibilidad y habilidad para entender a la ciudad dentro del ámbito técnico (Bobbio, 2004). La formación de Giovannoni es fundamental para comprender su postura respecto a la conservación de las ciudades, en virtud de que en esa época, en Italia era excepcional tener una formación que combinara las ciencias con las humanidades, dada la rígida división disciplinar que existía entre técnicos e intelectuales. De ahí que su visión le permitiera traspasar las fronteras entre la arquitectura, el arte y la historia junto con la ingeniería civil, así como con la tecnología de la construcción y el higienismo (Zucconi, 2014).

Como resultado de esta formación, Giovannoni buscó intervenir la ciudad de una manera integral, pues al estar convencido de la necesidad de realizar demoliciones y así proveer de una mayor funcionalidad e higiene a las ciudades, argumentó que la estrategia para alcanzar dicho objetivo debía guiarse hacia la reducción del volumen bajo el principio del llamado adelgazamiento (Bobbio, 2004).

La teoría del adelgazamiento que propuso Giovannoni surge como una respuesta ante su oposición a las demoliciones indiscriminadas, puesto que él estaba consciente de que había que liberar ciertos espacios por cuestiones de salud pública y de circulación, de ahí que se dio a la tarea de elaborar ciertas directrices para intervenir la ciudad antigua, bajo un enfoque más sensible respecto a la valorización de los viejos edificios, puesto que para él la ciudad era concebida como el lugar ideal para combinar lo antiguo, a través de la conservación, con las nuevas construcciones a fin de compaginar el arte y la técnica (Zucconi, 2014).

En 1925, Giovannoni publicó su obra Cuestiones de Arquitectura, en la cual dedica un capítulo a la llamada arquitectura ambiental, argumentando que el entorno de los monumentos constituye "un patrimonio vasto y diversificado que debe ser considerado como un 'monumento colectivo' por reconocer, preservar e interpretar" (Stabile, 2017, p. 135), lo que resulta ser una respuesta ante la incertidumbre que surge respecto a cómo debía ser manejada la relación entre la ciudad antigua y la ciudad moderna, esto lo llevó a valorar ya no solo el monumento como objeto singular, sino al conjunto como una entidad (Sánchez, 2017).

Si bien la historia urbana suele hacer referencia a los ejemplos de las ciudades europeas, es importante señalar que el incipiente reconocimiento de la ciudad histórica también tuvo eco en América. Ryberg-Webster y Kinahan (2014) mencionan que la preservación urbana en Estados Unidos se remonta al siglo XIX, cuando los llamados conservacionistas llevaron a cabo los primeros trabajos de salvaguarda de sitios asociados a la fundación de la nación, acompañada de trabajos de embellecimiento, así como mejorar las condiciones de vida en las nacientes ciudades industrializadas (Birch y Roby, 1984). Así mismo, las primeras acciones que podrían considerarse de conservación urbana ocurrieron en la década de los treinta, cuando se creó un distrito histórico en Charleston, Carolina del Sur, producto de la llamada zonificación propia de la planificación moderna de ciudades.

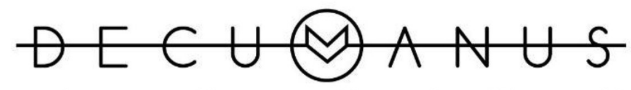

REVISTA INTERDISCIPLINARIA SOBRE ESTUDIOS URBANOS 
Sin duda, la segunda mitad del siglo XIX y principios del Xx fueron testigos de múltiples voces que aclamaban el reconocimiento de los cascos antiguos, ante las evidentes amenazas de la inaplazable modernización urbana. No debe olvidarse que estas voces no surgen de manera espontánea, sino que son una respuesta a las diversas obras que se emprendieron en aras de la idealizada modernización, lo cual implicó trastocar la llamada ciudad antigua.

\section{Haussmann y la intervención de París como detonador de la conservación urbana}

Hasta ahora se han revisado diversas posturas desarrolladas por diferentes urbanistas y pensadores en cuanto al reconocimiento de la ciudad antigua y su encuentro con la modernidad, las cuales deben ser leídas como una respuesta a los primeros estragos de la modernidad y el progreso, que comenzaron a materializarse a través de reformas urbanas sin precedentes, convirtiéndose en una amenaza directa para los conjuntos antiguos.

Las diversas intervenciones realizadas en la ciudades europeas durante dicho periodo, van más allá de un mero capricho modernizador, pues debe tomarse en cuenta que si bien el llamado urbanismo moderno comienza a consolidarse tanto en la escuela alemana e inglesa, como en la norteamericana, existe una realidad urbana caracterizada por la insalubridad de las ciudades, situación que se va a evidenciar aún más con las epidemias de cólera que se extendieron no solo en Europa, sino también en Asia, África y América (González, Casanova y Pérez, 2011).

La insalubridad que permeaba a las ciudades se hizo más evidente en los núcleos de las urbes antiguas, puesto que estaban sobrepoblados, provocando que en ellos se presentaran altos indices de enfermedades, lo que obligó a las autoridades a intervenir dichos espacios, que generalmente estaban rodeados por murallas, mismas que fueron demolidas a fin de liberar espacios y adecuar las vialidades (Zucconi, 2014).

Entre los ejemplos más relevantes y polémicos se encuentra la intervención de París durante el siglo XIX, encabezada por Georges-Eugène Haussmann (1809-1891), quien emprendió el proyecto de adecuación de la ciudad lo que provocó la alteración de barrios antiguos (Gaja, 1996; Choay, 2009), a fin de dotar a París de vías de comunicación más eficientes y mejorar las condiciones de salubridad (Sánchez, 2008, p. 27; Chueca Goitia, 1985, p. 161). Sin embargo, antes de emitir un juicio, se debe entender el momento histórico que se vivía, pues comenzaba a asomarse un urbanismo incipiente que tenía como ideal la urbe moderna, y a la vieja ciudad aún no se le reconocía su carácter de monumento. No obstante, el acto no pasó inadvertido, sino que provocó un primer enfrentamiento entre los que podrían llamarse los destructores modernizadores y los conservadores (Chateloin, 2008).

La imagen de París se vio trastocada $y$, por ende, el imaginario que sus habitantes tenían de su ciudad, sin duda, estos serán los primeros en percatarse de dicha transformación camaleónica, lo que a su vez, tendrá un reflejo en

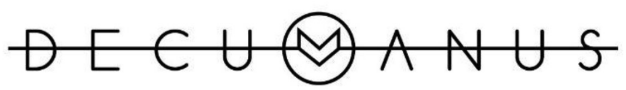

REVISTA INTERDISCIPLINARIA SOBRE ESTUDIOS URBANOS 
la literatura, por ejemplo, Charles Baudelaire (1821-1867) en su obra Las flores del mal, dedica a Víctor Hugo (18021885) el poema "El Cisne", en el que expresa la nostalgia por lo perdido, "El viejo París ya no existe más (la forma de la ciudad cambia más rápido jay! Que el corazón de un mortal" (Baudelaire, 1868, p. 66). Estas palabras, inspiradas en las transformaciones que estaba sufriendo la ciudad, surgieron en respuesta a los textos de Víctor Hugo, que asemejaban una alegoría al futuro prometedor, publicados en la Paris Guide (1867) y en Les Misérables (1862); es fácil percibir que Víctor Hugo denota en su obra una nostalgia por el París que guardaba en su memoria -dado que vivía en el exilio- y al mismo tiempo delata una confianza hacia el futuro que vendría acompañado de una organización territorial y urbana coherente (Calatrava, 2016, p. 64).

Ahora bien, la transformación que experimentó París fue sin duda una intervención sin precedentes, el proyecto de modernización implicó la modificación del sistema viario preexistente y la demolición de inmuebles para dar lugar a los grandes bulevares; al respecto las autoridades parisinas estaban totalmente conscientes de los efectos que estas obras implicaban, puesto que buscaron la forma de conservar la memoria de aquellas que estaban por desaparecer. En 1851, la administración del Segundo Imperio convocó a la Comisión de Monumentos Históricos para elaborar el estudio fotográfico de las edificaciones que por su antigüedad requirieran trabajos de restauración, mediante la contratación de fotógrafos que documentaran el proceso del antes y el después de la intervención. No obstante, se debe resaltar que los trabajos de documentación efectuados por dichos fotógrafos sufrieron cierta manipulación, a fin de justificar las demociones. Al respecto, Anna Lovecchio (2005) realizó un exhaustivo análisis de las fotografías realizadas por Charles Marville (1813-1879), uno de los encargados de dicha misión.

Lovecchio señala que las fotografías reflejan una intención, puesto que el punto de vista del fotógrafo, en cuanto a la posición de la cámara, tiene la intención de enfatizar calles sinuosas, estrechas y sórdidas, lo que se logra al ubicar la cámara a poca distancia del suelo, evitando posicionar al fotógrafo al centro de la vía, sino de lado, a fin de modificar y acortar la perspectiva (Lovecchio, 2005), lo que tendría como resultado una imagen deformada de la realidad, soporte válido para justificar los trabajos de demolición.

Las obras emprendidas por Haussmann en París tuvieron un efecto dominó en diversas ciudades europeas. En Alemania se practicó el aislamiento de monumentos, principalmente catedrales. En Italia se llevó a cabo la ampliación de Florencia en 1864, en la cual se buscó sistematizar la traza medieval con nuevas calles en ángulo recto, para así promover el aislamiento de monumentos; en Roma se perforaron nuevas avenidas (Glendinning, 2013).

Diversas propuestas de transformación urbana se produjeron en otras ciudades; en el caso de Viena y Barcelona, los cascos antiguos no se vieron afectados, como sucedió con el París haussmaniano. En lo que se refiere a Barcelona, François Choay menciona que el ensanche respetó la ciudad me-

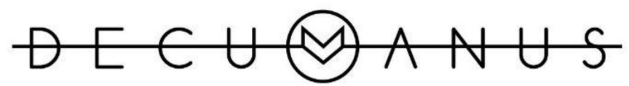

REVISTA INTERDISCIPLINARIA SOBRE ESTUDIOS URBANOS 
dieval, en contra de los deseos de IIdefonso Cerdá (1815-1876), a diferencia de Otto Wagner (1841-1918), quien en su proyecto de Plan Regulador para la Ciudad de Viena, menciona que "Conviene aquí respetar la belleza y satisfacer las exigencias de salubridad y de circulación por medio de una conservación adecuada del patrimonio existente, procurando aportar las mejoras propias que satisfagan las exigencias modernas" (Choay, 2009), puesto que su propuesta buscaba generar un diálogo entre la ciudad antigua y la moderna, bajo el precepto de que las nuevas formas contemporáneas no debían contraponerse al legado del pasado (Layuno, 2014, p. 104).

\section{Reflexiones finales}

Todas estas visiones, algunas de ellas sincrónicas, aunadas a otras más, fueron conformando el andamiaje de una disciplina dirigida a la conservación del entorno urbano y a la diferenciación no solo estética de una parte de la ciudad, sino también poseedora de un funcionamiento particular que debía ser atendido de una manera diferente a su contraparte moderna. Es evidente que este proceso no surge de manera aislada, sino que es producto de una época y de una ideología que provocó enfrentamientos entre aquellos que idealizaron la llegada de la modernidad versus los intelectuales defensores y admiradores de las edificaciones y conjuntos históricos.

Difícilmente puede imaginarse este proceso sin la revolución ideológica que provocó arribo de la modernidad, que venía acompañada no solo de una manera diferente de concebir la ciu- dad, sino que trajo nuevas formas de construir que en su momento fueron sobrevaloradas respecto a las edificaciones erigidas en épocas pasadas, generando un dualidad entre pasado y presente, entre progresistas y conservadores, que a pesar de los enfrentamientos discursivos, se alcanzó un consenso que se plasmó justo en el reconocimiento de la ciudad histórica, pues el mismo proceso de expansión urbana permitió que los ideales de progreso se materializaran en las zonas aledañas a los cascos antiguos.

Por lo tanto, será justo la postura que se tome hacia el pasado, respecto a su gestión, lo que dará lugar a la diversificación de los discursos, si bien la identificación y el reconocimiento de su valor es el primer paso, la siguiente acción deriva en su conservación como vestigio del pasado y de la memoria; esto, por supuesto, es lo que va a generar posturas encontradas respecto a cuál debe ser el destino de dichos espacios con base en las necesidades de su tiempo.

En ese sentido debe tomarse en consideración toda la serie de cambios que experimentó la sociedad en la época, en donde existía un anhelo por reflejar progreso, pero también había una necesidad por adaptar las ciudades a nuevas condiciones a fin de solucionar problemas de habitabilidad, como fueron todos aquellos relativos a la salubridad, lo que condujo a la modificación y demolición de diversas estructuras, cuyo efecto se vio reflejado en la alteración de los conjuntos históricos a los cuales aún no se les reconocía ningún valor o, en caso contrario, su condición de viejos los hizo ver como elementos sustituibles.

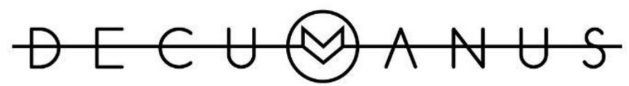

REVISTA INTERDISCIPLINARIA SOBRE ESTUDIOS URBANOS 
A través de este recorrido histórico es interesante destacar cómo la necesidad por salvaguardar el pasado, si bien comenzó a manifestarse mediante acciones aisladas, fueron encontrando eco en otras latitudes, condición determinante para respaldar el naciente movimiento de conservación urbana, puesto que los discursos a finales del siglo XIX y principios del XX se fueron multiplicando, con ello se sentaron las bases respecto a cómo debían ser tratados los conjuntos históricos ante la inminente avalancha de cambios que acompañaron la llegada del llamado progreso e industrialización de las ciudades.

Por consiguiente, será a partir del surgimiento de esta incipiente preocupación por reconocer el valor histórico de las ciudades, que comienza una segunda historia que reflejará la maduración respecto al tema, así como el notorio involucramiento de autoridades y organismos internacionales comprometidos por salvaguardar el patrimonio urbano, lo que se verá reflejado en una serie de documentos que han ido guiando hasta nuestros días la valoración y protección del patrimonio.

Asimismo, debe resaltarse la importancia de tener una lectura de la época para poder entender este proceso desde una perspectiva más amplia, pues los cambios que se dan en el ámbito social, económico, político y sobre todo ideológico, tienen un inminente efecto en el espacio urbano, pues como se mencionó, la segunda mitad del siglo xIX representó un parteaguas en la ideología occidental, cuando la gran ansiedad por ser modernos, se convirtió en una navaja de doble filo, dado que la moderni- dad tuvo diversos efectos, provocando múltiples críticas por parte de los pensadores de la época, pues si bien hubo quienes se manifestaron por dar rienda suelta al progreso a costa de borrar las huellas del pasado, otros más concebían los vestigios como objetos irremplazables y de un valor único que debían ser protegidos, de ahí que el movimiento de conservación, como lo menciona Glendinning (2013), es inequívocamente un hijo de la modernidad, misma que debe ser leída desde sus diferentes aristas, no como un fenómeno homogéneo, en tanto que las manifestaciones de esta no fueron solo con un matiz progresista y demoledor del pasado, sino que despertó y se conformó una nueva disciplina: la conservación urbana.

\section{Referencias}

Bandarin, F., y van Oers, R. (2012). The Historic Urban Landscape. Managing heritage in an urban century. Oxford: Wiley y Blackwell.

Baudelaire, C. (1868). Las flores del mal (2006. ${ }^{a}$ ed.). Argentina: Editorial del Cardo.

Bobbio, R. (2004). La questione della città antica. Recuperado de https:// architettura.unige.it/per/doc/bobbior/020301.pdf

Calatrava, J. (2016). El París de Haussmann como territorio de la utopía: Victor Fournel (1865) y Victor Hugo (1867). Quintana. Revista de Estudios del Departamento de Historia da Arte, 15, 53-71.

Capel, H. (2002). La morfología de las ciudades. Barcelona: Ediciones del Serbal.

Capitel, A. (1988). Metamorfosis de monumentos y teorías de la restauración. Madrid: Alianza. 
Castells, M. (2004). La cuestión urbana

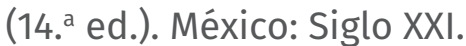

Chateloin, F. (2008). El centro histórico. ¿Concepto o criterio en desarrollo? Arquitectura y urbanismo, XXXIX(2-3), 10-23.

Choay, F. (2009). El reino de lo urbano y la muerte de la ciudad (S. Urrieta, Trad.). Andamios, 6(12).

Chueca Goitia, F. (1985). Breve historia del urbanismo (10. ${ }^{\mathrm{a}}$ ed.). Alianza.

De la Corte Carmona, J. (2015). Schumpeter y la destrucción de instituciones por los innovadores. Universidad Pontificia Comillas, Madrid.

Descartes, R. (2010). Discurso del Método. Madrid: Austral -Espasa.

Di Montauto, B. (Ed.). (2003). Arte collezionismo conservazione. Scritti in onore di Marco Chiarini. Giunti Editore.

Díaz Berrio, S. (2007). Protección y rehabilitación del patrimonio cultural urbano. México: Universidad Autónoma Metropolitana.

Fernández Christlieb, F. (1998). La influencia francesa en el urbanismo de la ciudad de México: 1775-1910. En México Francia: Memoria de una sensibilidad común siglos XIX-XX (Vol. 1). México: Centro de estudios mexicanos y centroamericanos.

Freestone, R. (2007). The Internationalization of the City Beautiful. International Planning Studies, 12(1), 21-34, https:// doi.org/10.1080/13563470701346527

Gaja, F. (1996). Las teorías sobre la intervención urbanística en la ciudad preindustrial. Universitat Politècnica de València.

Gellner, E. (1997). Antropología y política. Revoluciones en el bosque sagrado. Barcelona: Gedisa.

Glendinning, M. (2013). The conservation movement: A history of architectural preservation: Antiquity to modernity. Nueva York: Routledge.

González, L., Casanova, M., y Pérez, J. (2011). Colera. Historia y actualidad. Revista de Ciencias Médicas de Pinar del Río, 15(4). Recuperado de http://scielo.sld. cu/scielo.php?script=sci_arttext\&pi$d=S 1561-31942011000400025$

González, M. (1994). Medievo y Renacimiento, ¿Ruptura o continuidad? (El marco historiográfico de una polémica). The Spanish Journal for Medieval Philosophy, 1, 9-26.

Harvey, D. (1998). La condición de la posmodernidad. Investigación sobre los orígenes del cambio cultural. Buenos Aires: Amorrortu Editores.

Layuno, Á. (2013). Las primeras “ciudades de la industria": Trazados urbanos, efectos territoriales y dimensión patrimonial. La experiencia de Nuevo Baztán. Scripta Nova: Revista Electrónica de Geografia y Ciencias Sociales, XVII(451). Recuperado de http://www. ub.edu/geocrit/sn/sn-451.htm\#_edn27

Layuno, Á. (2014). Procesos y proyectos de configuración estética del espacio urbano. La Viena de Otto Wagner. Arte y Ciudad-Revista de Investigación, 5. Recuperado de file:///Users/luzcrodriguezsanchez/Downloads/Dialnet-ProcesosYProyectosDeConfiguracionEsteticaDelEspaci-4838475.pdf

Le-Duc, V. (1995). Restauración (Diccionario Razonado de Arquitectura) (M. De la Piedra, Trad.). Cuaderno de Notas, 4, 15-36.

Lefebvre, H. (1988). De la ciudad a la sociedad urbana. En M. Bassols, R. Donoso, y A. Méndez (Eds.), Antología de la sociología urbana (pp. 463-478). México: Universidad Nacional Autónoma de México.

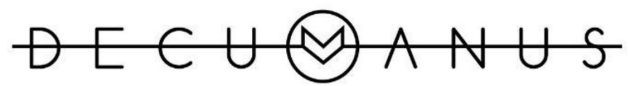

REVISTA INTERDISCIPLINARIA SOBRE ESTUDIOS URBANOS 
Lovecchio, A. (2005). Parisian Visions: Haussmann, Marville and Meryon. Recuperado de Tufts University: http:// emerald.tufts.edu/programs/mma/fah189a/alovecchio/Parisian_Visions.pdf

Marx, C. (2003). El 18 Brumario de Luis Bonaparte. Madrid: Fundación Federico Engels.

Pérez Blázquez, A. (2010). El cambio de mentalidad colectiva: Renacimiento, humanismo, reforma y contrareforma. Proyecto Clío, (36).

Prats, L. (2000). El concepto de patrimonio cultural. Cuadernos de antropología social, 11, 115-136.

Pugin, W. (1898). Contrast: Or a Parallel Between the Noble Edifices of the Middle Age, and Corresponding Buildings of the Present Day; Shewing the Present Decay of Taste. Recuperado de https:// ia800401.us.archive.org/33/items/contrastsorparal00pugi/contrastsorparal00pugi.pdf

Rielg, A. (1987). El culto moderno a los monumentos. Madrid: Visor distribuciones.

Ruskin, J. (1996). Las siete lámparas de la arquitectura. Ediciones Coyoacán.

Ryberg-Webste, S., y Kinahan, K. (2014). Historic Preservation and Urban Revitalization in the Twenty-first Century. Journal of Planning Literature, 29(2), 119-139. https://doi.org/10.1177/0885412213510524

Sánchez, A. (2017). Las problemáticas actuales en los centros históricos. Gentrificación y viralización. Esempi di Architecttura, 2.

Sánchez de Juan, J.-A. (2000). La “Destrucción creadora": El lenguaje de la reforma urbana en tres ciudades de la Europa Mediterránea a finales del siglo XIX (Marsella, Nápoles y Barcelona). Scripta Nova. Revista Electrónica de Geografía y Ciencias Sociales, (63). Recuperado de http://www.ub.edu/geocrit/sn-63.htm
Sánchez, G. (2008). Planeación moderna de ciudades. México: Trillas.

Schumpeter, J. (1996). Capitalismo, socialismo y democracia. Barcelona: Ediciones Folio.

Sitte, C. (1926). Construcción de ciudades según principios artísticos (E. Canosa, Trad.). Recuperado de https://archive. org/details/QArm121

Stabile, F. (2017). Gustavo Giovannoni e la cultura dell'ambientismo. Bolletino del Centro di Studi per la Storia dell'Architettura, 1, 135-146.

Stübben, J. (1907). City Building (2008. ${ }^{a}$ ed.). Recuperado de https://urbanism. uchicago.edu/page/joseph-st\%C3\%BCbbens-city-building

Tomaselli, F. (Ed.). (2013). Restauro Anno Zero. Il varo della prima Carta italiana del restauro nel 1882 a seguito delle proteste internazionali contro la falsificazione della Basilica di San Marco a Venezia. ARACNE editrice.

Zeayter, H., y Mansour, A. (2018). Heritage conservation ideologies analysis - Historic urban Landscape approach for a Mediterranean historic city case study. Housing and Building National Research Center Journal, 14, 345-356. https://doi.org/10.1016/j.hbrcj.2017.06.001

Zucconi, G. (2014). Gustavo Giovannoni: A Theory and a Practice of Urban Conservation. Change Over Time, 76-91. https://doi.org/10.1353/cot.2014.0009 
DECUOANHS

REVISTA INTERDISCIPLINARIA SOBRE ESTUDIOS URBANOS
Núm. 4. Vol. 4. Octubre 2018-Octubre 2019.

Instituto de Arquitectura, Diseño y Arte.

Universidad Autónoma de Ciudad Juárez. ISSN: 2448-900X 